golimumab and certolizumab) and all 3 had recurrence of the lesions, in 2 patients the anti-TNF was replaced by a non-anti-TNF biological. Topical treatment was used in all cases, one patient also required systemic treatment with methotrexate. Conclusions: TNF antagonist induced psoriasis is a well-described adverse event. Pustular psoriasis is the most frequent presentation. In most cases there is no personal or family history of psoriasis. Topical therapy may be effective but some patients require discontinuation of the drug. Skin lesions can reappear when switching to another anti-TNF drug.

Disclosure of Interest: None declared

DOI: 10.1136/annrheumdis-2017-eular.2877

\section{AB1209-HPR THE PREVALENCE OF DENTAL AND SINUS INFECTION IN PATIENTS WITH RHEUMATOID ARTHRITIS BEFORE BIOLOGIC THERAPY INITIATION: USEFULNESS OF A SYSTEMATIC SCREENING?}

C. Tawil, E. Descamps, M. Forien, A. Gardette, E. Palazzo, S. Ottaviani, P. Dieudé. Rheumatology Department, Bichat Hospital, Paris, France

Background: Introduction of the biologic therapies has dramatically improved the outcome of severe rheumatoid arthritis (RA). Biologic therapies play a central role in the control of synovial inflammation. However they also decrease host defenses leading to an increased rate of infection. Because of their adverse effects, a careful assessment is needed before their initiation. A systematic assessment of dental or sinus infection before a biologic therapy is not required.

Objectives: The aim of our study was to assess the prevalence and the usefulness of a systematic screening of oral (dental and/or sinus) infection of RA patients before biologic therapy initiation.

Methods: This was a monocentric retrospective study. We included RA (ACR/EULAR 2010 criteria) patients with active disease despite disease-modifying anti-rheumatic drugs (DMARDs) and requiring biologic therapy initiation between 2010 and 2016. The following parameters were collected: demographic and disease characteristics, disease activity (C-reactive protein, disease activity score (DAS) 28), currents therapies (DMARDS, corticosteroids). Dental infection was assessed by stomatologist after clinical and panoramic dental X-ray evaluation. Sinusitis was defined on sinus computed tomography as partial or complete opacification of one or more sinus cavities. Factors associated with oral infections were analyzed in uni- and multivariate models.

Results: We included 223 RA patients $(79.4 \%$ of female, mean \pm SD disease duration of $8.9 \pm 8.6$ years). The mean age was $54 \pm 10.9$ years, $70.8 \%$ rheumatoid factor (RF) positive, $84.4 \%$ anti-citrullinated protein antibody (ACPA) positive and $68.1 \%$ had radiographic damages. The mean DAS 28 was $5.5 \pm 2.6 ; 71 \%$ of patients received corticosteroids (mean $7 \mathrm{mg}$ per day of equivalent prednisone) and $63 \%$ methotrexate (mean $17.8 \mathrm{mg}$ per week). No patient had pain or other sinus or dental symptoms. Before biologic agent initiation, systematic dental and sinus screening revealed an oral infection in $31.5 \%$ of patients (dental: $20.2 \%$ and sinus: $14.8 \%$ ). In univariate analysis, active smoking was associated with a higher risk of oral infection $(\mathrm{OR}=2.16[1.02-4.57], \mathrm{p}=0.038)$ and methotrexate with $a$ lower rate $(O R=0.43$ [0.23-0.81], $p=0.006)$. Corticosteroid, disease duration, DAS 28, RF, ACPA and structural damages were not associated with oral infection. No significant association was confirmed with oral infection using multivariate analysis.

Conclusions: In our study, one third of RA patients requiring biologic agents had asymptomatic oral infection. The high prevalence of oral infection in RA patients suggests the usefulness of systematic dental and sinus screening before biologic therapy initiation.

Disclosure of Interest: None declared

DOI: 10.1136/annrheumdis-2017-eular.4037

\section{AB1210-HPR MENDELIAN RANDOMIZATION ANALYSIS INDICATES SERUM URATE HAS A CONDITIONAL CAUSAL EFFECT ON SERUM CREATININE AND RENAL FUNCTION}

J. Liu ${ }^{1}$, H. Zhang ${ }^{1}$, Z. Dong ${ }^{1}$, J. Zhou ${ }^{1}$, Y. Ma ${ }^{1}$, Y. Li ${ }^{1}$, Q. Qian ${ }^{1}$, Y. Yang ${ }^{1,2}$, X. Wang ${ }^{1,2}$, H. Zou ${ }^{3,4}$, L. Jin ${ }^{1,2}$, J. Wang ${ }^{1,2,4}$. ${ }^{1}$ State Key Laboratory of Genetic Engineering, Collaborative Innovation Center for Genetics and Development, School of Life Sciences, Fudan university, Shanghai; ${ }^{2}$ Fudan-Taizhou Institute of Health Sciences, Taizhou; ${ }^{3}$ Division of Rheumatology, Huashan Hospital, Fudan University; ${ }^{4}$ Institute of Rheumatology, Immunology and Allergy, Fudan university, Shanghai, China

Background: Uric acid, the weak organic acid and the end product of purine nucleotide degradation, is excreted predominantly by the proximal tubules [1]. Although large numbers of epidemiological, molecular and animal studies have focused on various pathogenic effects of serum uric acid, inclding chronic kidney disease (CKD), metabolic syndrome, and coronary artery disease [2], whether the serum uric acid is an independent risk factor or has causal impact on serum creatinine ( $\mathrm{SCr}$ ) and renal function remains unclear.

Objectives: We aim to study the effect of serum uric acid on renal function by applying the method of Mendelian randomization.

Methods: The study was represented by estimated glomerular filtration rate (eGFR) with potential confounding factors in 3,734 Chinese subjects. Four genetic variants of uric acid transporter genes (rs1481012 [ABCG2], rs16890979
[SLC2A9], rs2231137 [ABCG2] and rs3799352 (SLC17A1]) were selected for this study as they had highest correlation with serum uric acid in Chinese population in our previous study. In this research, serum uric acid was selected as exposure, genetic risk score of uric acid transporters was selected as instrumental variable, and SCr and eGFR were selected as the outcomes.

Results: 1) The results of the analysis showed that increased serum uric acid has a causal effect on reducing estimated glomerular filtration rate in both female population and the subjects who were under 65 years old. Because of protective effects on renal function of ovarian hormones such as estrogen, we postulated that estrogen might be the cause leading to the difference between men and women. 2) We also found that increased serum uric acid led to the damage of renal function in the subjects with normal eGFR value. 3) In addition, the serum uric acid was a risk factor to renal function in the subjects with relative high level of fasting glucose or who were smoking currently. Because of metabolic defects in people affected by diabetes, renal glucose reabsorption was increased, thus further sustaining hyperglycemia in patients.

Conclusions: Serum urate has causal effects on renal dysfunction in either female or individuals of under 65 , or normal eGFR, or high level of fasting glucose, or current smokers.

References:

[1] Pasalic D, Marinkovic N, Feher-Turkovic L. Biochem Medica 2012;22(1):63-75. [2] Sakhaee K. J Nephrol 2014;27(3):241-5.

Acknowledgements: National Natural Science Foundation of China (31521003), Science and Technology Committee of Shanghai Municipality (11DJ1400102), International S\&T Cooperation Program of China (2013DFA30870), Ministry of Science and Technology (2011BAI09B00), 111 Project (B13016), and Program for 2012 Outstanding Medical Academic Leader for Hejian Zou. Computational support was provided by the High-End Computing Center located at Fudan University.

Disclosure of Interest: None declared

DOI: 10.1136/annrheumdis-2017-eular.5601

\section{AB1211-HPR THE USE OF SUBCUTANEOUS METHOTREXATE IN POLISH PATIENTS WITH RHEUMATOID ARTHRITIS}

M. Przygodzka ${ }^{1}$, K. Sikorska-Siudek ${ }^{1}$, R. Radomski ${ }^{2}$, S. Bojanowski ${ }^{2}$. ${ }^{1}$ Mazovian Centre of Rheumatology and Osteoporosis; ${ }^{2}$ iEHR.eu, Warsaw, Poland

Background: Methotrexate (Mtx) should be the drug of the first choice in rheumatoid arthritis (RA) if there are no contraindications to use it. The efficacy of $\mathrm{Mtx}$ is measured by remission or low disease activity and depends on the dose taken. Higher doses (25-30 mg/week) are more effective, but intolerance is the main cause of discontinuation of oral treatment. Subcutaneous Mtx is efficient alternative in those cases.

Objectives: The aim of this study was to evaluate subcutaneous Mtx use frequency in Polish patients and change oral form for subcutaneous as well.

Methods: The disease activity was assessed by Disease Activity Score 28 (DAS 28 ) during the first visit (V1) and after 3 months therapy (V2) and compliance with therapy as well.

Results: There were 194 RA patients diagnosed by the ACR and 1997 and/or ACR/EULAR 2010 criteria. 144 patients were treated by oral Mtx (group A) and 50 patients (group B) by subcutaneous Mtx at the time of study enrolment (V1).37 patients of group $A(26 \%)$ required changes in therapy during V2, $24(17 \%)$ were switched to subcutaneous Mtx (group A1). 6 patients of group B (12\%) required change of treatment during V2, including 2 patients (4\%) with subcutaneous Mtx, who were switched to oral Mtx.The main cause of changing therapy from oral to subcutaneous was gastrointestinal intolerance of high dose of Mtx. 69 patients $(12 \%)$ of group A required additional steroid therapy compared to $18(36 \%)$ of group B. Average DAS 28 decreased by 0.58 in group A1 in oral treatment and during the subcutaneous treatment time decreased by next 0.23 . In group A1 during oral treatment 14 (58\%) patients used $25 \mathrm{mg} /$ week and 20 patients (83\%) used $25 \mathrm{mg} /$ week during subcutaneous treatment time.

Conclusions: Patients treatment by oral Mtx often require modification of therapy in comparison to patients treated by subcutaneous Mtx, including more frequent use of steroids

The main cause of oral intolerance are ailments of the digestive system.

Change of oral to subcutaneous therapy allows administration of higher doses of Mtx and results in decrease of DAS28 in comparison to the patients continuing their oral treatment.

Disclosure of Interest: None declared

DOI: 10.1136/annrheumdis-2017-eular.6963

\section{AB1212-HPR DYNAMICS OF ARTICULAR SYNDROME IN RHEUMATOID ARTHRITIS AGAINST CORRECTION OF PROGESTERONE INSUFFICIENCY}

M. Salokhiddinov, A. Ahmedov. Rheumatology and Osteopathy, Tashkent Medical Academy, Tashkent, Uzbekistan

Background: According to the literature, sex hormones manifest themselves as immune modulators that inhibit the ones and stimulate other immune functions. Studies have shown that patients with RA, decrease of estradiol and particularly 
progesterone in the blood serum is found in the women of reproductive age. According to the literature, progesterone has a close relationship with T-cell immunity. A value of T-cell deficiency plays an important role in the cause of the autoimmune process

Objectives: To study of progesterone correction in RA patients. The aim of this study was to evaluate the effectiveness of progesterone deficiency correction in patients with rheumatoid arthritis based on the analysis of articular syndrome Methods: The study involved 40 patients with a documented diagnosis of RA, women of reproductive age. The study group consisted of 30 patients who had correction of progesterone deficiency background in the pathogenetic therapy. The control group consisted of 10 female patients who are on the pathogenetic therapy. We used the following criteria: disease activity and severity of the articular syndrome with determining of the number of tender and swollen joints, indices of DAS and HAQ. Statistical analysis of the results of research used the method of parametrics of Student criterion

Results: Among the patients studied disease duration ranged from 3 months to 19 years (mean age $10 \pm 7,2$ years), 20 patients $(50 \%)$, from 5 to 10 years - $15(37.5 \%)$, and more than 10 years - 5 patients $(12.5 \%)$. X-ray picture of joint damage in the majority of patients corresponded to stage $2-3-87.5 \%$ (35 patients). Less common muscular disorders $(7.5 \%)$, mainly in patients taking long-term GC, myalgia and muscle malnutrition were detected in $7.5 \%$ and $25 \%$ of patients, respectively. In addition to the articular syndrome, the most frequent complaints of general weakness $(75 \%)$, irritability, sleep disorders and attention $(50 \%)$, anxiety and anxiety $(75 \%)$, low-grade temperature $(10 \%)$

Conclusions: Progesterone deficiency correction in RA patients against pathogenic therapy has improved performance of articular syndrome and improves quality of life, such as vitality, general health and social function as well as it contributes to the positive dynamics of mental health

References:

[1] McInnes IB, Schett G. The pathogenesis of rheumatoid arthritis New Engl. J. Med. 2011;365:2205-19.

[2] Wells G et al. Validation of the 28-joint Disease Activity Score (DAS28) and European League Against Rheumatism response criteria based on C-reactive protein against disease progression in patients with rheumatoid arthritis, and comparison with the DAS28 based on erythrocyte sedimentation rate. Ann. Rheum. Dis. 2009;68:954-60.

[3] Ware JE, Sherbourne CD. The MOS 36-Item short-form health survey. Med. Care. Med Care. 1992;30(6):473-83.

Disclosure of Interest: None declared

DOI: 10.1136/annrheumdis-2017-eular.1306

\section{AB1213-HPR A NATIONAL RHEUMATOID ARTHRITIS REGISTRY SUPPORTED BY A PUBLIC POLICY AS A STRATEGY FOR DISEASE CONTROL AND RISK MANAGEMENT IN COLOMBIA}

O. Valencia ${ }^{1}$, P. Sánchez ${ }^{1}$, L. Acuña ${ }^{1}$, L. Soler ${ }^{1}$, C.E. Toro ${ }^{2} .{ }^{1}$ Cuenta de Alto Costo; ${ }^{2}$ Asociación Colombiana de Reumatología, Bogotá, Colombia

Background: Rheumatoid arthritis (RA) is a chronic disease that implies high direct and indirect costs for the health system $(1,2)$. According to the needs of the health care system, the clinical interests and the national regulatory framework, it was developed a national registry information of RA patients (3).

Objectives: To show how a registry of information that would meet the RA situation was developed and to present the results obtained from the analysis of the Registry on this first year.

Methods: A national RA Registry was created after a comprehensive literature review to identify the relevant variables to determine monitoring indicators used by health insurers and health services providers in the attention of patients with RA. Variables were selected and defined by an agreement with clinical experts, thematic and methodological experts and were evaluated by the Ministry of Health in order to review and approve the structure to gather the information.

Results: A structure of 89 variables contained was defined. All entities must report annually to the Registry all the patients with a diagnosis of RA, their clinical and demographic characteristics, and the process of care and costs (3). On its first year the Registry provided a baseline of the disease situation of 68.357 patients with RA. Prevalence, incidence, state of disease and drugs including synthetic and biologic DMARDs were analyzed (Table 1). Most important results were: mean age 57 years; relation women: men 5.2:1; age at onset of disease: 36 years, mean evolution time of disease: 7 years; population with DAS 28 measured 45.6\%; mean DAS-28 2.8; percentage of the patient with DMARD therapy $78.9 \%$ and bDMARD $16.5 \%$.

Table 1. Characteristics of the population

\begin{tabular}{lccccc}
\hline Variable & Obs & Mean/prop. & Std. Dev. & Min & Max \\
\hline Age & 68376 & 57.0 & 13.3 & 22 & 104 \\
Age at onset of disease & 13607 & 36.4 & 7.7 & 20 & 51 \\
Evolution time of RA & 37963 & 7.5 & 8.1 & 0 & 51 \\
DAS28 & 30956 & 2.82 & 1.37 & 0 & 1 \\
"Women & 57461 & 84.04 & & & \\
"Men & 10914 & 15.96 & & & \\
"bDMARD & 11276 & 16.50 & & & \\
"DMARD & 53374 & 78.01 & & & \\
\hline
\end{tabular}

Conclusions: A national Registry supported by an official policy, with data from the real world provided for healthcare insurers gives an opportunity to obtain a global vision and to identify failures and strengths in the attention process of $\mathrm{RA}$, and to develop indicators to obtain better outcomes. In the future, through continuous efforts towards improving the quality of care provided, these will allow to monitoring and decreasing the burden of RA in the country and may serve as a model to other countries.

References:

[1] Muñetón GA, Quintana G. La epidemiología de la artritis reumatoide. Rev Colomb Reumatol. 2015 Sep [cited 2016 Jul 25];22(3):145-7. Available from: http://www.elsevier.es/es-revista-revista-colombiana-reumatologia-374articulo-la-epidemiologia-artritis-reumatoide-S0121812315000845.

[2] Quintana G., Restrepo JP, Cáceres H, Rueda JD. Economic evaluation of the treatment of rheumatoid arthritis with anti-TNF biological therapy in Colombia. Acta Médica Colomb. 2011;36(1):24-9. Available from: http://www.scielo.org.co/pdf/amc/v36n1/v36n1a05.pdf.

[3] MSPS. Resolucion No. 1393. 2015. p. 14.

Disclosure of Interest: None declared

DOI: 10.1136/annrheumdis-2017-eular.5017

\section{AB1214-HPR SPINAL BRUCELLOSIS: A RETROSPECTIVE STUDY OF 30 CASES}

S. Rekik, S. Boussaid, I. Abdelkafi, S. Hela, I. Cheour, M. Elleuch. Rheumatology, la Rabta, Tunis, Tunisia

Background: Brucellosis is a major health problem in Mediterranean countries including Tunisia. The clinical presentation of this zoonosis varies considerable but osteoarticular involvement and spinal brucellosis particulary is the commonest complication.

Methods: This study was carried out between 2000 and 2015. One hundred and six patients with infectious spondylodicitis hospitalized in the department of rheumatology were analyzed. All patients were thoroughly interrogated subjected to a rigorous clinical examination and a battery of investigations including: complete blood count, urine analysis, blood culture, erythrocyte sedimentation rate, $\mathrm{C}$ - reactive protein (CRP) and serology for brucellosis. The imaging of spine ordered including: X-ray, bone scan and magnetic resonance imaging (MRI) with contrast enhancement.

Results: Thirty of the 106 patients $(28 \%)$ proved to have spinal brucellosis. The mean age of these patients was 53 years (range 15-68 years) and female/men ratio at 1.5 . The mean delay of diagnosis was 8 months. The following symptoms were observed: fever in 14 patients and back pain in all of patients. Other symptoms were less frequently observed, such as splenomegaly (2 patients), peripheral adenopathy ( 5 patients) and diarrhea (2 patients). Laboratory exams showed elevated erythrocyte sedimentation rate in 13 patients, high levels of CRP in all patients and leukocytosis in 11 cases. Wright serology was positive in 21 of the patients. Brucella melitensis was isolated in blood cultures in 2 cases. Standard X-rays were performed in all patients. They showed signs of spondylodiscitis in the lumbar spine in 20 cases, cervical in 2 cases and dorsal in 8 cases. Ct-scan and MRI confirms the diagnosis and showed associated epiduritis in 2 cases or abscess in 6 cases. Bone biopsy with histopathological examination was performed in 7 cases. A combination of cycline and rifampicin was given to all patients. The duration of therapy was between 6 and 8 weeks.

Conclusions: Brucellosis is present with various clinical signs in endemic areas and may simulate many diseases. The need for prompt diagnosis and treatment of spinal brucellosis is of utmost importance to prevent serious bone destruction and severe neurologic Sequelae.

Disclosure of Interest: None declared

DOI: 10.1136/annrheumdis-2017-eular.6301

\section{HPR interventions (educational, physical, social and psychological)}

\section{AB1215-HPR EFFECTS OF BEETROOT JUICE SUPPLEMENTATION N ENDOTHELIAL FUNCTION AND MARKERS OF INFLAMMATION AMONG PATIENTS WITH RHEUMATOID ARTHRITIS}

A. Stavropoulos ${ }^{1}$, E. Manthou ${ }^{2}$, T. Nakopoulou ${ }^{3}$, K. Georgakouli ${ }^{2}$, A. Jamurtas ${ }^{2} .{ }^{1}$ Carnegie School of Sport, Leeds Beckett University, Leeds, United Kingdom; ${ }^{2}$ School of Physical Education and Sports Science, University of Thessaly; ${ }^{3}$ Radiology, General Hospital of Trikala, Trikala, Greece

Background: Nitric oxide (NO) is central in the process of vasodilatation (1). Limited bioavailability of NO often associates with endothelial dysfunction, a precursor to atherogenesis (1). Such dysfunction is often observed in patients with chronic conditions such as Rheumatoid Arthritis (RA) (2) and Chronic Obstructive Pulmonary Disease (COPD) (3). Common therapies for this include the administration of nitrate-rich medication. However, in the general population beetroot juice supplementation has been shown to increase NO bioavailability (4). It could therefore have beneficial effects on endothelial function of these patients as well. 